\section{Commentary: An innovative strategy for expanding the donor pool}

\author{
Oliver K. Jawitz, MD, MHS, and \\ Carmelo Milano, MD
}

Heart transplantation continues to be constrained by a limited supply of donor organs. In an effort to expand the US donor pool, the use of allografts from donation after circulatory death (DCD) donors is currently being investigated. Early reports have estimated that the adoption of DCD heart transplantation may lead to an increase in overall heart transplant volume by as much as $30 \%$. ${ }^{1,2}$ Indeed, our center has seen a recent increase in volume of approximately $30 \%$, largely due to the use of DCD donors. Furthermore, DCD heart transplantation has been performed in the United Kingdom and Australia for several years, with reported early outcomes comparable to traditional donation after brain death transplantation. ${ }^{3,4}$

In this issue of the Journal, Smith and colleagues ${ }^{5}$ from New York University Langone Health report outcomes from their 8-patient feasibility pilot study of DCD heart transplantation using cardiopulmonary bypass (CPB) for normothermic regional perfusion (NRP). Unlike the direct procurement and perfusion approach, which does not permit an in situ functional evaluation of the donor organ and relies on a potentially cost-prohibitive ex vivo perfusion platform, NRP permits rapid reperfusion and assessment of allograft function under physiologic conditions. Although NRP DCD heart transplantation protocols are already being implemented internationally, most notably by Papworth Hospital in the United Kingdom, ${ }^{6}$ the protocol under investigation by the New York University group is novel with regard to the use of full CPB with left ventricular venting, antemortem heparinization, donor withdrawal in the operating room, and co-location of

\footnotetext{
From the Division of Cardiovascular and Thoracic Surgery, Department of Surgery, Duke University Medical Center, Durham, NC.

Disclosures: The authors reported no conflicts of interest.

The Journal policy requires editors and reviewers to disclose conflicts of interest and to decline handling or reviewing manuscripts for which they may have a conflict of interest. The editors and reviewers of this article have no conflicts of interest.

Received for publication Aug 2, 2021; revisions received Aug 2, 2021; accepted for publication Aug 3, 2021; available ahead of print Aug 8, 2021

Address for reprints: Oliver K. Jawitz, MD, MHS, Department of Surgery, Duke University School of Medicine, Box 3443, Durham, NC 27710 (E-mail: oliver.jawitz@ duke.edu).

J Thorac Cardiovasc Surg 2022;164:571-2

$0022-5223 / \$ 36.00$

Copyright (C) 2021 by The American Association for Thoracic Surgery

https://doi.org/10.1016/j.jtcvs.2021.08.007
}

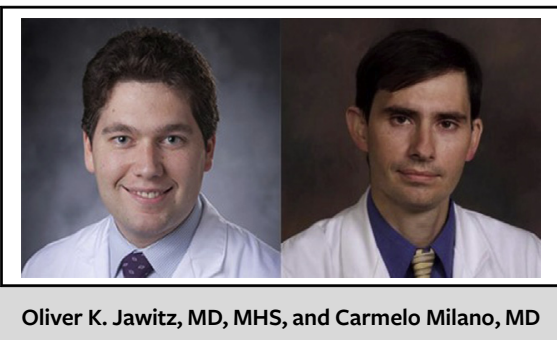

CENTRAL MESSAGE

Early outcomes after DCD heart transplantation with NRP appear promising; however, several logistical, financial, and ethical hurdles must be overcome before broad implementation is feasible.

donor and recipient. Overall, the outcomes demonstrated by Smith and colleagues ${ }^{5}$ are extremely encouraging, including the ability to wean all donor hearts from CPB without inotropes, a $100 \%$ recipient survival with a median follow-up of approximately 1 year, an average postdischarge left ventricular ejection fraction of $64 \%$, and no patients requiring mechanical circulatory support. The authors also report the utility of NRP for safe recovery of abdominal organs.

Before DCD heart transplantation with NRP can be more widely adopted, several important hurdles must be overcome. First, NRP with CPB as described by the investigators is logistically complex and requires significant effort and coordination by the donor hospital, procuring teams, perfusionists, and the Organ Procurement Organization. Acceptance of the protocol by all stakeholders is a prerequisite for successful implementation. Second, protocols requiring donor-recipient co-location are unlikely to be broadly applicable. A US NRP protocol will need to be developed involving longer distance cold static storage or ex vivo perfusion, keeping in mind the cost implications of the latter. Last, and perhaps most important, DCD heart procurement with NRP raises important ethical issues that have yet to be fully addressed, with certain countries such as Australia disallowing the practice. ${ }^{7}$ Ethical concerns have focused on the practice of clamping the arch head vessels before initiation of CPB as an active step to maintain brain death. ${ }^{7}$ On the other hand, advocates of the practice emphasize that the use of these organs fulfills the last 
benevolent wish of the deceased and their family. Although the debate surrounding the ethical aspects of NRP heart procurement is ongoing, it is of paramount importance that clear communication takes place with the donor family to avoid misunderstandings, because maintenance of trust from the donating public is at stake.

Early indicators suggest that DCD heart transplantation has the potential to make heart transplantation a feasible option for significantly more patients with heart failure. Further investigation is necessary to determine whether DCD heart procurement with NRP versus direct procurement and ex vivo reperfusion is viable and advantageous.

\section{References}

1. Messer S, Page A, Rushton S, Berman M, Tsui S, Catarino P, et al. The potential of heart transplantation from donation after circulatory death donors within the United Kingdom. J Heart Lung Transplant. 2019;38:872-4.
2. Jawitz OK, Raman V, DeVore AD, Mentz RJ, Patel CB, Rojers J, et al. Increasing the United States heart transplant donor pool with donation after circulatory death. J Thorac Cardiovasc Surg. 2020;159:e307-9.

3. Messer S, Page A, Axell R, Berman M, Hernández-Sánchez J, Colah S, et al. Outcome after heart transplantation from donation after circulatory-determined death donors. J Heart Lung Transplant. 2017;36:1311-8.

4. Chew HC, Iyer A, Connellan M, Scheuer S, Villanueva J, Gao L, et al. Outcomes of donation after circulatory death heart transplantation in Australia. J Am Coll Cardiol. 2019;73:1447-59.

5. Smith DE, Kon ZN, Carillo JA, Chen S, Gidea CG, Piper GL, et al. Early experience with donation after circulatory death heart transplantation using normothermic regional perfusion in the United States. J Thorac Cardiovasc Surg. 2022; 164:557-68.e1.

6. Messer SJ, Axell RG, Colah S, White PA, Ryan M, Page AA, et al. Functional assessment and transplantation of the donor heart after circulatory death. J Heart Lung Transplant. 2016;35:1443-52.

7. American College of Physicians Board of Regents. Ethics, determination of death, and organ transplantation in normothermic regional perfusion (NRP) with controlled donation after circulatory determination of death (cDCD): American College of Physicians statement of concern. April 17, 2021. https://www.acponline.org/acp_policy/policies/ethics_determination_of_death_ and_organ_transplantation_in_nrp_2021.pdf 